\title{
Miniature Transparent UWB Antenna with Tunable Notch for Green Wireless Applications
}

\author{
T. Peter ${ }^{*(1)}$, Y.Y.Sun ${ }^{(2)}$, H.F. AbuTarboush ${ }^{(1)}$, R. Nilavalan ${ }^{(1)}$ and S.W. Cheung $^{(2)}$ \\ (1) Dept of Electronics and Computer Engineering, Brunel University, Kingston Lane, Uxbridge, UB8 3PH, UK \\ Email:thomas.peter@brunel.ac.uk \\ (2)Dept of Electrical and Electronic Engineering, The University of Hong Kong, Pokfulam Road, Hong Kong \\ Email:yysun@eee.hku.hk
}

\begin{abstract}
A miniature transparent UWB antenna with tunable notch that can be incorporated with a solar panel for harnessing solar energy has been proposed and presented. The antenna demonstrates a good omni-directional radiation pattern throughout the FCC bandwidth of $3.1-10.6 \mathrm{GHz}$ and a comparable gain making it a good candidate for future green wireless applications.
\end{abstract}

\section{INTRODUCTION}

In recent years, work on transparent antennas have been actively pursued[1-2]. However, other than the few papers that have been occasionally published, not many papers have surfaced. This is because of the lossy nature of the transparent material and the low efficiency and the difficulty in fabricating the designs. One earlier work on a transparent monopole antenna using AgHT-4 showed improved efficiency with a trade-off in transparency [1]. A much more recent work on a coplanar waveguide (CPW) ultra wideband (UWB) antenna showed a $20 \%$ improvement to efficiency using a novel soldering technique and using the same material while maintaining full transparency "in press" [2]. One of the factors affecting the efficiency is the surface resistance of the AgHT-8 material which actually increases and reduces the conductivity as the size of the antenna is miniaturized causing further radiation losses besides the usual losses at the connectors due to soldering and impedance mismatch. In this paper, a miniature UWB AgHT-8 antenna design as shown in Fig. 1 is proposed and presented. It demonstrates almost a close but lesser efficiency than in [2] whilst giving a very good nearly omni-directional pattern throughout the entire FCC UWB band of 3.1 GHz to $10.6 \mathrm{GHz}$. The antenna also demonstrated a very wide bandwidth and comparable peak gain.

The antenna too, can be incorporated with a tunable notch as in [3] to filter out the $5 \mathrm{GHz}$ frequency band so as not to cause interference to the existing $5 \mathrm{GHz}$ WLAN application.

The miniature, slim and transparent feature of this antenna allows it to be integrated to a mini solar panel or cell for harnessing solar energy to provide backup power for efficient management and use of the battery in UWB compact and slim devices. This has been demonstrated using a mini solar panel attached to a digital display later on in the paper. Such solar powered transparent antennas make them a good candidate for the green wireless technology of the future.

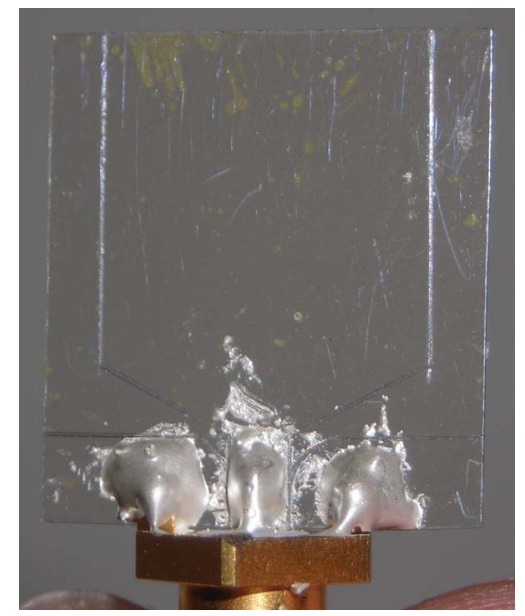

Fig. 1 Miniature transparent UWB Antenna without slot for notch tuning. 


\section{ANTENNA DESIGN}

The miniature transparent UWB antenna is designed and fabricated using AgHT- 8 for both the monopole and the $50 \Omega$ CPW feed and ground planes. The AgHT- 8 film is $0.175 \mathrm{~mm}$ thick and has a surface resistance of $8 \Omega$-Sq which is equivalent to a conductivity $(\sigma)$ of $1.25 \times 10^{5} \mathrm{~S} / \mathrm{m}$ [1]. The AgHT-8 film is made up of a coat of transparent silver layer sandwiched between two layers of tin oxide on a thin polyethylene terephthalate (PET) substrate with a relative permittivity of 3.24. The antenna is designed and optimized as an upright rectangle with bevels at the bottom end and fed by a $50 \Omega \mathrm{CPW}$ line as illustrated in Fig. 2. The bevel angles are optimized to give the best impedance matching [4]. The bevel edge and the top ground edge basically act as capacitors and varying the capacitance helps improve the impedance matching and thus the return loss. The chamfers further help to improve the bandwidth by bringing the return loss curve below the $-10 \mathrm{~dB}$ line even more. Introducing a $0.3 \mathrm{~mm}$ slot at a distance of $1 \mathrm{~mm}$ along each vertical edge of the radiator like in [3] can produce a tuning notch to filter out any unwanted frequency band within the obtained bandwidth. Maximizing the length of the slot will produce a notch at the lower frequency while minimizing the length of the slot will produce a notch at the upper frequency. The slots are demonstrated in Fig. 3.

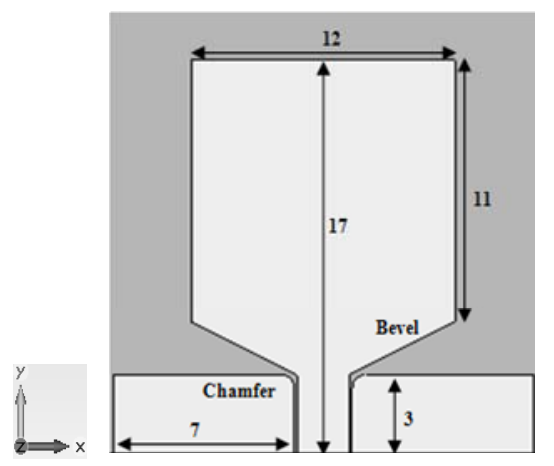

Fig. 2 Layout of the UWB Transparent Antenna

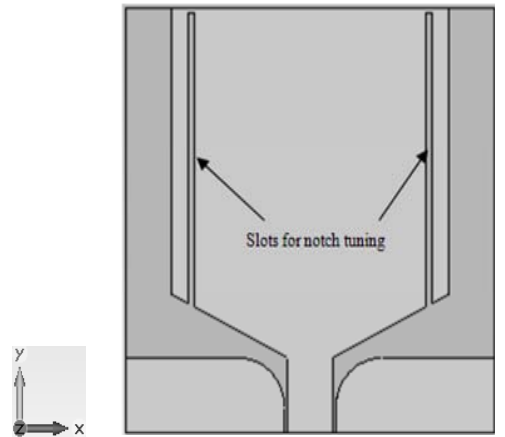

Fig. 3 Transparent UWB Antenna with Slots for notch tuning

\section{MEASUREMENT AND SIMULATION RESULTS}

The transparent polymer UWB antenna was designed and simulated on CST Microwave Studio. The performance of the proposed antenna was verified by fabricating and testing the transparent UWB antenna and measuring the return loss. Results of the simulated and measured return loss are shown in Fig. 4 and 5. The measured return loss show better performance than that of the simulated. This improvement can be attributed to the shape of the chamfer during fabrication. The measured return loss shows a $-10 \mathrm{~dB}$ level response from $3 \mathrm{GHz}$ onwards.

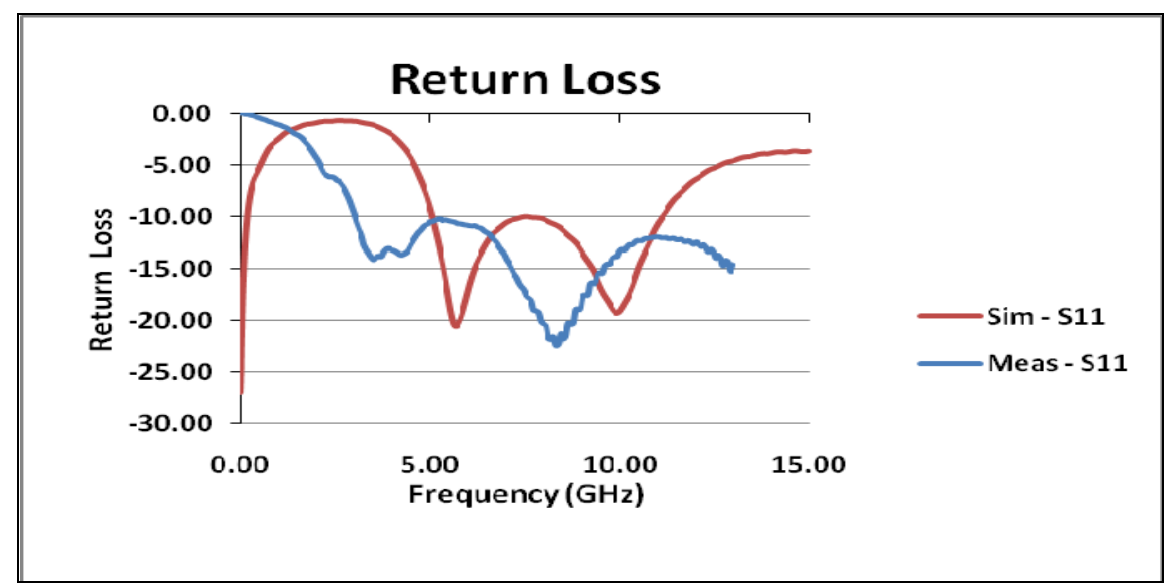

Fig. 4 Simulated and measured return loss of the miniature transparent UWB antenna 


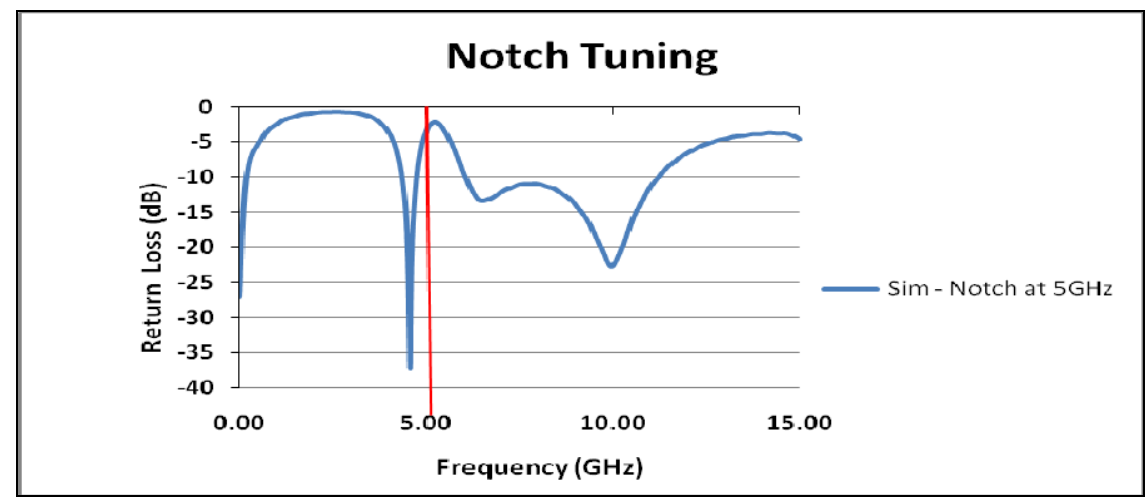

Fig. 5. Simulated return loss of the miniature uwb with a notch at $5 \mathrm{GHz}$

Efficiency, gain and radiation pattern measurements of the fabricated prototype tested on Satimo's radiation pattern measurement equipment, StarLab further affirmed the findings based on the return loss. The measured peak gain and efficiency are shown in Fig. 6 and Fig. 7. The measured 3-D radiation patterns of the antenna as shown in Fig. 8 show a very good omni-directional pattern at the 3,5,7 and $9 \mathrm{GHz}$ frequencies which makes this antenna suitable for receiving signal from all direction throughout the FCC stipulated bandwidth of $3.1-10.6 \mathrm{GHz}$.

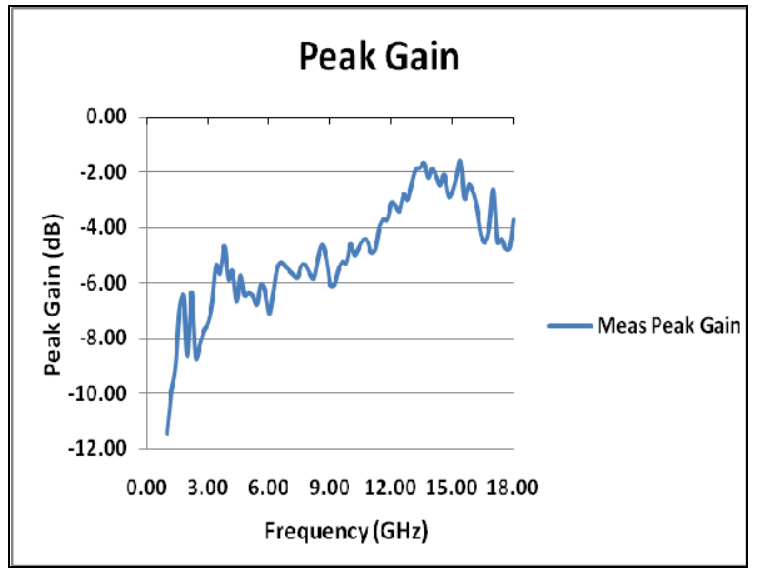

Fig. 6. Measured peak gain

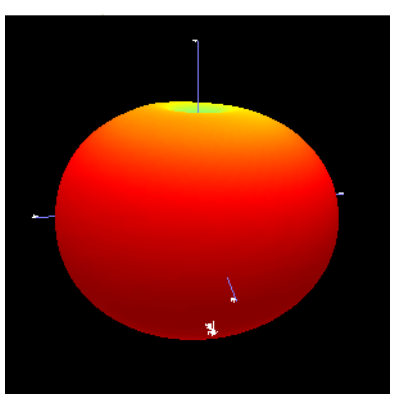

a)

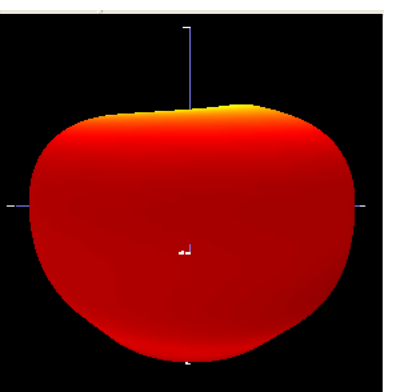

b)

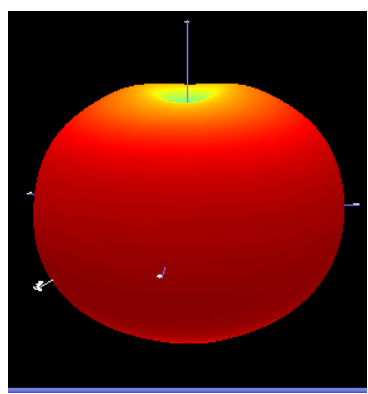

c)

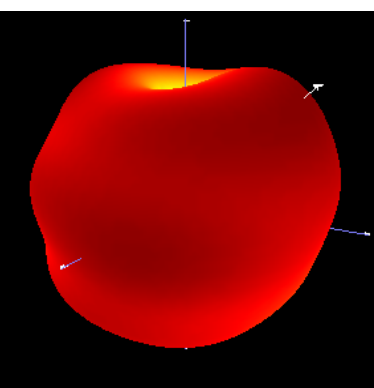

d)

Fig. 8 Measured 3-D radiation patterns of the antenna: a) $3 \mathrm{GHz}$ b) $5 \mathrm{GHz}$ c) $7 \mathrm{GHz}$ d) $9 \mathrm{GHz}$ 


\section{INTEGRATION OF SOLAR PANEL}

Integrating a miniature solar panel to the back of the transparent UWB antenna enables solar rays to be harnessed through the transparent surface of the antenna to produce solar energy to provide back-up power for efficient management of the batteries in devices in which this type of antenna can be employed. Fig. 9 further illustrates this.

A digital display is powered on to display a zero when the light from the room's lighting falls onto the mini solar panel from TRONY Solar (Model: 1025I). The display slowly powers off when the solar panel is obstructed or blocked from the lighting. This solar panel also works in dim lighting and hence makes the integrated antenna suitable for indoor wireless applications.

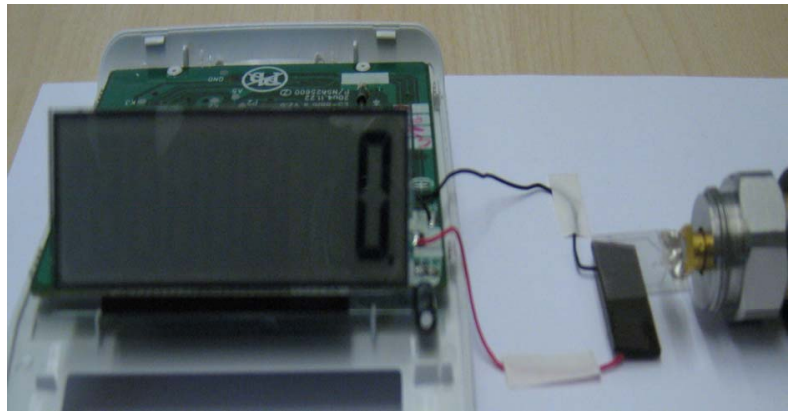

a)

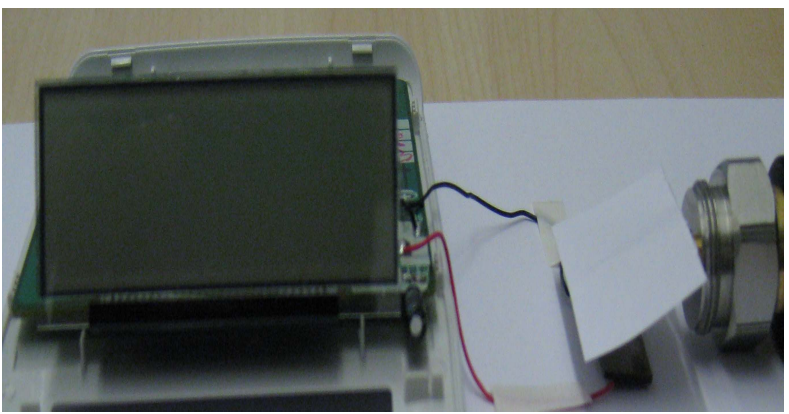

b)

Fig.9 a) Display being powered on when solar panel is not blocked b) Display being powered off when the solar panel is blocked

\section{CONCLUSIONS}

A miniature transparent ultra wideband antenna that can be integrated with solar panels for solar energy harvesting or providing back up power for wireless application batteries has been proposed and demonstrated. Although the efficiency is quite low probably as mentioned earlier due to higher resistance being encountered on the AgHT-8 with miniaturization in experiment, the 3-D patterns indicate a very nearly omni-directional pattern that is suitable for ultra wideband requirements. Increasing the size of this antenna design may increase the efficiency and could be used with larger solar panels for harvesting greater amounts of energy for various use in wireless applications or even buildings. Work on these are still ongoing and results are expected to be published in later publications.

\section{ACKNOWLEDGEMENTS}

The authors wish to acknowledge CPFilms, Portsmouth, UK for their support in providing the AgHT-8 film used in this research.

\section{REFERENCES}

[1] Hyok Jae Song; Tsung Yuan Hsu; Sievenpiper, D.F.; Hui Pin Hsu; Schaffner, J.; Yasan, E., "A Method for Improving the Efficiency of Transparent Film Antennas," Antennas and Wireless Propagation Letters, IEEE, vol.7, pp.753-756, 2008.

[2] T.Peter, R. Nilavalan, H.F. Abu Tarboush, S.W. Cheung, “A Novel Technique and Soldering Method To Improve Performance Transparent Polymer Antennas" (Accepted for publication in Antennas and Wireless Propagation Letters - August 2010).

[3] Su Won Bae, Hyung Kuk Yoon, Woo Suk Kang, Young Joong Yoon, Cheon-Hee Lee, “A Flexible Monopole Antenna with Band-notch Function for UWB Systems", Proceedings of the Asia-Pacific Microwave Conference 2007.

[4] M. J. Ammann, "Control of the impedance bandwidth of wideband planar monopole antennas using a beveling technique,"Microwave Optical Technology Letters, vol.30(4), pp. 229-232, 2001. 